\title{
ON THE SMALLER PERTURBATIONS OF THE LUNAR
}

\section{ARGUMENTS*}

BY

\section{ERNEST WILLIAM BROWN}

The problem of finding the effects of planetary action on the motion of the moon has not yet been satisfactorily solved. As far as periodic terms are concerned the works of HILL, $\nmid$ NEwcomb $\ddagger$ and RADAU $\S$ have tended to considerably shorten and simplify the calculation, reducing it, when once the disturbing function has been computed, to some simple substitutions in a general formula. But the method is not available for finding the non-periodic changes produced on the mean motions of the perigee and node. I propose to obtain here some simple formulæ which permit these perturbations to be found quickly and with all the accuracy that can be desired. The results will be applied to the effects produced by planetary action and by the figure of the earth, but certain of the constants whose values are given below are available for the calculations of any non-periodic perturbations of the perigee and node. The notation adopted here is, in general, that of my paper "On the formation of the derivatives of the lunar coördinates with respect to the elements" $\|$; the investigation below is, in fact, a continuation of that paper to a certain extent.

\section{(i) Formulae for finding the non-periodic perturbations of the perigee and node.}

1. The motion of the moon under the attractions of the earth and the sun considered as particles is supposed to have been found. The coördinates are thus expressed in trigonometric series of the angles $w_{4}, w_{5}, w_{6}$ and an angle not

* Presented to the Society February 27, 1904. Received for publication February 26, 1904.

$\dagger$ On certain lunar inequalities due to the action of Jupiter, Washington Astronomical Papers, vol. 3 (1891), pp. 373-393.

$\ddagger$ Action of the Planets on the Moon, Washington Astronomical Papers, vol. 5 (1894), pp. 101-295.

¿ Recherches concernant les inégalités planétaires du mouvement de la lune, A n nal es de l'Observatoire de Paris (Mémoires), vol. 21 (1892), pp. 1-114.

|| Transactions of the American Mathematical Society, vol. 4 (1903), pp. 234248. This paper will be referred to below by the letter $\mathrm{F}$. 
containing the constants of integration, with coefficients depending on constants of integration $c_{1}, c_{2}, c_{3}$ and known constants. The angles $w_{j}$ are of the form

$$
w_{4}=b_{4} t+\epsilon, \quad w_{5}=b_{5} t+\pi, \quad w_{6}=b_{6} t+\theta,
$$

where $b_{4}=n$, is the mean motion and $b_{5}, b_{6}$ are the mean motions of the perigee and node; the $b_{i}$ are functions of the $c_{i}$ and known constants; $\epsilon, \pi, \theta$ are the other three constants of integration. The $c_{i}, w_{j}$ are called below the "elements" of the moon's motion.

If a new small disturbing function $\lambda R$ be added, the resulting changes in lunar elements are obtained by solving the equations (F., art. 18)

$$
\frac{d c_{i}}{d t}=\lambda \frac{\partial R}{\partial w_{i+3}}, \quad \frac{d w_{j}}{d t}=-\lambda \frac{\partial R}{\partial c_{j-3}}+b_{j} \quad(i=1,2,3 ; j=4,5,6) .
$$

In all practical cases squares of $\lambda$ may be neglected so that the undisturbed values of the elements may be substituted in the derivatives of $R,-$ a fact which enables us to substitute for $R$ its constant term when $R$ is expanded in a trigonometric series, for we are only concerned here with the constant parts of $d w_{j} / d t$. We therefore use the letter $R$ henceforward to denote the constant term of that function.

The equations (1) can now be solved and they give

$$
c_{i}=\text { const. }, \quad w_{j}=-\lambda t \frac{\partial R}{\partial c_{i-3}}+b_{j} t+\text { const. }
$$

The theory is at first supposed to be expressed, not in terms of $w_{j}, c_{i}$, but in terms of $w_{j}$ and $n, c_{2}, c_{3}$, the semi-canonical system (F., art.10): $c_{2}$ contains the square of the eccentricity constant as a factor, and $c_{3}$ that of the inclination constant. Let $\delta n, \delta c_{2}, \delta c_{3}$ be the changes in $n, c_{2}, c_{3}$ caused by $\lambda R$ and $\delta b_{i}$ the changes in the $b_{j}$. Then, since $b_{4}=n$,

$$
\begin{aligned}
& \delta b_{4}=-\lambda \frac{\partial R}{\partial c_{1}}+\delta n, \\
& \delta b_{5}=-\lambda \frac{\partial R}{\partial c_{2}}+\frac{\partial b_{5}}{\partial n} \delta n+\frac{\partial b_{5}}{\partial c_{2}} \delta c_{2}+\frac{\partial b_{5}}{\partial c_{3}} \delta c_{3}, \\
& \delta b_{6}=-\lambda \frac{\partial R}{\partial c_{3}}+\frac{\partial b_{6}}{\partial n} \delta n+\frac{\partial b_{6}}{\partial c_{2}} \delta c_{2}+\frac{\partial b_{6}}{\partial c_{3}} \delta c_{3} .
\end{aligned}
$$

The old definition of the letter $n$ is to be retained, that is, it is to represent the mean motion; thus $\delta b_{4}=0$ and $\delta n=\lambda \partial R / \partial c_{1}$. We shall, for the present, define $c_{2}, c_{3}$ to be the same as when $\lambda=0$, so that $\delta c_{2}=0, \delta c_{3}=0$. These 
definitions are possible because $c_{1}, c_{2}, c_{3}$ are constant whether $\lambda$ is zero or not. Hence

$$
\delta b_{5}=-\lambda\left(\frac{\partial R}{\partial c_{2}}-\frac{\partial b_{5}}{\partial n} \frac{\partial R}{\partial c_{1}}\right), \quad \delta b_{6}=-\lambda\left(\frac{\partial R}{\partial c_{3}}-\frac{\partial b^{2}}{\partial n} \frac{\partial R}{\partial c_{6}}\right),
$$

where $b_{5}, b_{6}$ are expressed in terms of $n, c_{2}, c_{3}$ and $R$ is expressed in terms of $c_{1}, c_{2}, c_{3}$.

I have shown (F., no. 10) that

$$
-\frac{\partial b_{5}}{\partial n}=\frac{\partial c_{1}}{\partial c_{2}}, \quad-\frac{\partial b_{6}}{\partial n}=\frac{\partial c_{1}}{\partial c_{3}}
$$

where $b_{2}, c_{1}$ are expressed in terms of $n, c_{2}, c_{3}$. Hence

$$
\delta b_{5}=-\lambda \frac{\partial R}{\partial c_{2}}, \quad \delta b_{6}=-\lambda \frac{\partial R}{\partial c_{3}},
$$

where $R$ is now expressed in terms of $n c_{2}, c_{3}$.

These are the required expressions for $\delta b_{5}, \delta b_{6}$.

3 . The results $(2)$ are remarkable in that they show the greater simplicity obtained by using the semi-canonical system $n, c_{2}, c_{3}$ instead of the canonical system $c_{1}, c_{2}, c_{3} ;$ a similar result was found in another connection (F., art. 10). Moreover, the main difficulty in obtaining accuracy, namely, the formation of derivatives with respect to $n$, has disappeared. One or two transformations are all that are necessary in order to calculate $\delta b_{5}, \delta b_{6}$ with good accuracy from a numerical theory and with complete accuracy from a theory in which the numerical value of the ratio of the mean motions is substituted while the other constants are left in a literal form. The results of my theory are therefore immediately available when the derivatives of $R$ with respect to $c_{2}, c_{3}$ have been expressed in terms of derivatives with respect to $e, k$-the constants of the theory.

In the applications it is never necessary to go beyond the second powers of the eccentricities and inclinations, and all the functions considered are expansible in squares of these quantities and of the ratio of the parallaxes; the square of this latter ratio is negligible. As we have to form derivatives with respect to $c_{2}, c_{3}$, that is, with respect to $\mathrm{e}^{2}, \mathrm{k}^{2}$, it will be necessary, for this degree of accuracy, to find the expansions as far as the second powers and products of $\mathrm{e}^{2}, e^{\prime 2}, \mathbf{k}^{2}$.

4. We have, when the independent variables $b_{1}, c_{2}, c_{3}$ are changed to $b_{1}$, $\mathrm{e}^{2}, \mathrm{k}^{2}$

$$
\frac{\partial R}{\partial \mathrm{e}^{2}}=\frac{\partial R}{\partial c_{2}} \frac{\partial c_{2}}{\partial \mathrm{e}^{2}}+\frac{\partial R}{\partial c_{3}} \frac{\partial c_{3}}{\partial \mathrm{e}^{2}}, \quad \frac{\partial R}{\partial \mathrm{k}^{2}}=\frac{\partial R}{\partial c_{2}} \frac{\partial c_{2}}{\partial \mathrm{k}^{2}}+\frac{\partial R}{\partial c_{3}} \frac{\partial c_{3}}{\partial \mathrm{k}^{2}},
$$

where $\partial / \partial \mathbf{e}^{2}, \partial / \partial \mathbf{k}^{2}$ denote derivatives with respect to $\mathrm{e}^{2}, \mathbf{k}^{2}$. Since $c_{2}$ contains 
the factor $\mathrm{e}^{2}$, and $c_{3}$ the factor $\mathrm{k}^{2}$, we obtain from (2), on omitting the terms of an order higher than the second,

with a similar equation for $\delta b_{6}$.

$$
-\frac{\delta b_{5}}{\lambda}=\frac{\partial R}{\partial c_{2}}=\frac{\frac{\partial R}{\partial \mathrm{e}^{2}}}{\frac{\partial c_{2}}{\partial \mathrm{e}^{2}}}-\frac{\frac{\partial R}{\partial \mathrm{k}^{2}} \frac{\partial c_{3}}{\partial \mathrm{e}^{2}}}{\frac{\partial c_{2}}{\partial \mathrm{e}^{2}} \frac{\partial c_{3}}{\partial \mathrm{k}^{2}}},
$$

When $R, c_{2}, c_{3}$ are expanded in powers of $\mathrm{e}^{2}, e^{\prime 2}, \mathrm{k}^{2}$, in the form

$$
\begin{aligned}
R & =n^{2} a^{2}\left[R(0)+\mathrm{e}^{2} R\left(\mathrm{e}^{2}\right)+\mathrm{k}^{2} R\left(\mathrm{k}^{2}\right)+\cdots\right], \\
c_{2} & =n a^{2}\left[\mathrm{e}^{2} c_{2}\left(\mathrm{e}^{2}\right)+\mathrm{e}^{4} c_{2}\left(\mathrm{e}^{4}\right)+\mathrm{e}^{2} e^{\prime 2} c_{2}\left(\mathrm{e}^{2} e^{\prime 2}\right)+\cdots\right], \\
c_{3} & =n a^{2}\left[\mathrm{k}^{2} c_{3}\left(\mathrm{k}^{2}\right)+\mathrm{k}^{4} c_{3}\left(\mathrm{k}^{4}\right)+\mathrm{e}^{2} \mathrm{k}^{2} c_{3}\left(\mathrm{e}^{2} \mathrm{k}^{2}\right)+\cdots\right],
\end{aligned}
$$

where $R(0), \ldots, c_{2}\left(\mathrm{e}^{2}\right), \ldots, c_{3}\left(\mathbf{k}^{2}\right), \cdots$ are functions of $n^{\prime} / n$ only and therefore numerical coefficients, we obtain finally

$$
\begin{aligned}
-\frac{\delta b_{5}}{\lambda u}=\frac{R\left(\mathrm{e}^{2}\right)}{c_{2}\left(\mathrm{e}^{2}\right)} & {\left[1-\frac{2 \mathrm{e}^{2} c_{2}\left(\mathrm{e}^{4}\right)+\mathrm{k}^{2} c_{2}\left(\mathrm{e}^{2} \mathrm{k}^{2}\right)+e^{\prime 2} c_{2}\left(\mathrm{e}^{2} e^{\prime 2}\right)}{c_{2}\left(\mathrm{e}^{2}\right)}\right] } \\
- & \mathrm{k}^{2} \frac{R\left(\mathrm{k}^{2}\right) c_{3}\left(\mathrm{e}^{2} \mathrm{k}^{2}\right)}{c_{2}\left(\mathrm{e}^{2}\right) c_{3}\left(\mathrm{k}^{2}\right)}+\frac{2 \mathrm{e}^{2} R\left(\mathrm{e}^{4}\right)+\mathrm{k}^{2} R\left(\mathrm{e}^{2} \mathrm{k}^{2}\right)+e^{\prime 2} R\left(\mathrm{e}^{2} e^{\prime 2}\right)}{c_{2}\left(\mathrm{e}^{2}\right)},
\end{aligned}
$$

with a similar expression for $\delta b_{6}$ in which $c_{3}, c_{2}, \mathrm{k}$, e are respectively put for $c_{2}, c_{3}, \mathbf{e}, \mathbf{k}$.

These expressions are not completely accurate to the orders given owing to the final definitions of $e, k$. These constants are defined by means of the principal elliptic inequality and the principal term in latitude respectively. In general the periodic terms in $R$ will produce in the coefficients of these terms additional parts of the form $\lambda \alpha_{0} \mathrm{e}, \lambda \beta_{0} \mathrm{k}$ where $\alpha_{0}, \beta_{0}$ are numerical coefficients. In order to reduce to the final definitions it is therefore necessary to replace e by $\left(1-\lambda \alpha_{0}\right) \mathrm{e}, \mathrm{k}$ by $\left(1-\lambda \beta_{0}\right) \mathrm{k}$. The change in $\delta b_{2}$ is therefore

$$
-2 \frac{\partial b_{5}}{\partial e^{2}} \lambda \alpha_{0} \mathrm{e}^{2}-2 \frac{\partial b_{5}}{\partial k^{2}} \lambda \beta_{0} \mathrm{k}^{2}
$$

As $\partial b_{5} / \partial \mathbf{e}^{2}, \partial b_{5} / \partial \mathbf{k}^{2}$ are of the order $\left(n^{\prime} / n\right)^{2}$ at least, the orders of these additional parts relatively to the corresponding parts of order $\mathrm{e}^{2}, \mathbf{k}^{2}$ already found, are in the ratio $\left(n^{\prime} / n\right)^{2}: 1$. A similar remark holds in the case of $\delta b_{6}$. In no actual case do these portions give additions to the annual mean motions so great as $0 " .005$; they therefore can and will be neglected. These additional parts, however, do not violate the general theorem of this paper, namely, that algebraical developments in powers of $n^{\prime} / n$ are not required, for the coefficients $\alpha_{0}, \beta_{0}$ can be obtained without such developments $(F)$. 
5. The following numerical values are needed:

$$
\begin{aligned}
\frac{c_{2}}{n a^{2}} & =-.11845 \mathrm{e}^{2}-.02324 \mathrm{e}^{4}-.26363 \mathrm{e}^{2} \mathrm{k}^{2}-.00110 \mathrm{e}^{2} \mathrm{e}^{\prime 2} \\
\frac{c_{3}}{n a^{2}} & =-2.00206 \mathrm{k}^{2}-1.96376 \mathrm{k}^{4}-.28546 \mathrm{e}^{2} \mathrm{k}^{2}-.00568 \mathrm{e}^{\prime 2} \mathrm{k}^{2} \\
n & =17325594^{\prime \prime} ; \\
\mathrm{e} & =.10983, \quad \mathrm{k}=.044892, \quad e^{\prime}=.01677 .
\end{aligned}
$$

The values of $c_{2}, c_{3}$ have been found by the method given in F., art. 11 .

The relations between my constants and those $(e, \gamma)$ of DeLAunar's theory, though not needed here, may be given:

$$
\begin{aligned}
& \mathrm{e}=+2.00054 e-.36682 e^{3}-2.01160 e \gamma^{2}+.04873 e e^{\prime 2}, \\
& \mathrm{k}=+1.00013 \gamma-.49609 \gamma^{3}-.49924 \gamma e^{2}-.00037 \gamma e^{\prime 2} .
\end{aligned}
$$

(ii) Application to planetary action.

6. Let $x, y, z$ be the coördinates of the moon, $\xi, \eta, \zeta$ those of a planet referred to moving axes of $x, y$ in the plane of the ecliptic of which the $x$-axis points to the mean place of the sun, and an axis perpendicular to this plane. Then if $r^{2}=x^{2}+y^{2}+z^{2}, D^{2}=\xi^{2}+\eta^{2}+\zeta^{2}$ and $\lambda$ denotes the mass of the planet, we have, neglecting the ratio of the parallax of the sun to that of the moon,

$$
R=\frac{3}{2} \frac{(x \xi+y \eta+z \zeta)^{2}}{D^{5}}-\frac{1}{2} \frac{r^{2}}{D^{3}} .
$$

It will tend to simplicity if we neglect at the outset certain quantities whose squares are certainly negligible in the results; these are the solar eccentricity and the product of the inclinations of the planet and the moon to the ecliptic. As the constant term in $R$ is alone required we can further neglect the products $x y \xi \eta / D^{5}, x z \xi \zeta / D^{5}, y z \eta \zeta / D^{5}$. The function then can be put into the form:

$$
\begin{aligned}
R & =\frac{1}{4}\left(x^{2}+y^{2}-2 z^{2}\right)\left(\frac{1}{D^{3}}-\frac{3 \zeta^{2}}{D^{5}}\right)+\frac{3}{4}\left(x^{2}-y^{2}\right) \frac{\xi^{2}-\eta^{2}}{D^{5}} \\
& =\frac{1}{4}\left(u s-2 z^{2}\right)_{0}\left(\frac{1}{D^{3}}-\frac{3 \zeta^{2}}{D^{5}}\right)_{0}+\frac{3}{8}\left(u^{2}+s^{2}\right)_{0}\left(\frac{\xi^{2}-\eta^{2}}{D^{5}}\right)_{0}
\end{aligned}
$$

where $u=x+y \sqrt{-1}, s=x-y \sqrt{-1}$, and ()$_{0}$ denotes the constant term in the expansion of the included function. The separation of the parts depending on the coördinates of the moon from those depending on the coördinates of the planet is possible because when the solar eccentricity is neglected, they have no common angles. 
Let $n^{\prime}, a^{\prime}$, be the mean motion and mean distance of the sun; $n, a$, those of the moon ; $m^{\prime}$, the mass of the sun ; $m=n^{\prime} /\left(n-n^{\prime}\right)$; a, a certain constant which differs little from $a$;

$$
H=\frac{\lambda}{m^{\prime}}\left(\frac{a^{\prime 3}}{D^{3}}-\frac{3 \zeta^{2} a^{\prime 3}}{D^{5}}\right)_{0}, \quad K=\frac{\lambda}{m^{\prime}} a^{\prime 3}\left(\frac{\xi^{2}-\eta^{2}}{D^{5}}\right)_{0} ;
$$

then, since $\left(u^{2}\right)_{0}=\left(s^{2}\right)_{0}$, the expression for $R$ may be written

$$
\lambda R=\frac{\mathrm{m}^{2}}{(1+\mathrm{m})^{2}} \frac{\mathrm{a}^{2}}{a^{2}} n^{2} a^{2}\left[\frac{1}{4} H\left(\frac{u s-2 z^{2}}{\mathrm{a}^{2}}\right)_{0}+\frac{3}{4} K\left(\frac{u^{2}}{\mathrm{a}^{2}}\right)_{0}\right] .
$$

In this expression the only parts containing $\mathrm{e}, \mathrm{k}$ are the coefficients of $H, K$ within the square brackets. I already have the results for these since they were required in obtaining the motion in the main problem. I find

$$
\begin{aligned}
\left(\frac{u s-2 z^{2}}{\mathrm{a}^{2}}\right)_{0}=\cdots+.38229 \mathrm{e}^{2}-6.00851 \mathrm{k}^{2}+ & .0398 \mathrm{e}^{4} \\
& -3.0395 \mathrm{e}^{2} \mathrm{k}^{2}+.0053 \mathrm{k}^{4}+\cdots, \\
\left(\frac{u^{2}}{\mathrm{a}^{2}}\right)_{0}=\cdots+.25496 \mathrm{e}^{2}+.18030 \mathrm{k}^{2}- & .0142 \mathrm{e}^{4} \\
& -.4094 \mathrm{e}^{2} \mathrm{k}^{2}-.2035 \mathrm{k}^{4}+\cdots
\end{aligned}
$$

7. We must next calculate $H, K$. First, owing to the numerical values of the eccentricities, inclinations and masses of the planets, the only possible sensible effect of the eccentricities and inclinations is the inclination of Venus. Let then $V^{\prime \prime}, V^{\prime}$ be the mean longitudes of a planet and the sun, $a^{\prime \prime}, a^{\prime}$ their mean distances, $\gamma^{\prime \prime}$ the sine of half inclination of the planet's orbit to the elliptic, $h^{\prime \prime}$ the longitude of the planet's node. Then, omitting powers of $\gamma^{\prime \prime}$ beyond the second,

$$
\begin{aligned}
& \xi=-a^{\prime}+\left(1-\gamma^{\prime \prime 2}\right) a^{\prime \prime} \cos \left(V^{\prime \prime}-V^{\prime}\right)+\gamma^{\prime \prime 2} a^{\prime \prime} \cos \left(V^{\prime \prime}+V^{\prime}-2 h^{\prime \prime}\right), \\
& \left.\eta=\left(1-\gamma^{\prime \prime 2}\right) a^{\prime \prime} \sin \left(V^{\prime \prime}-V^{\prime}\right)-\gamma^{\prime \prime 2} a^{\prime \prime} \sin V^{\prime \prime}+V^{\prime}-2 h^{\prime \prime}\right) \text {, } \\
& \zeta=2 \gamma^{\prime \prime} a^{\prime \prime} \sin \left(V^{\prime \prime}-h^{\prime \prime}\right) ; \\
& D^{2}=a^{\prime 2}+a^{\prime \prime 2}-2 a^{\prime} a^{\prime \prime}\left(1-\gamma^{\prime \prime 2}\right) \cos \left(V^{\prime \prime}-V^{\prime}\right)-2 \gamma^{\prime \prime 2} a^{\prime} a^{\prime \prime} \cos \left(V^{\prime \prime}+V^{\prime}-2 h^{\prime \prime}\right) \\
& =D_{0}^{2}-2 \gamma^{\prime 2} a^{\prime} a^{\prime \prime}\left(V^{\prime \prime}+V^{\prime}-2 h^{\prime \prime}\right), \\
& \xi^{2}-\eta^{2}={a^{\prime}}^{2}-2 a^{\prime} a^{\prime \prime}\left(1-\gamma^{\prime \prime 2}\right) \cos \left(V^{\prime \prime}-V^{\prime}\right)+{a^{\prime \prime 2}}^{2}\left(1-2 \gamma^{\prime \prime 2}\right) \cos 2\left(V^{\prime \prime}-V^{\prime}\right) \\
& -2 \gamma^{\prime \prime 2} a^{\prime \prime}\left\{a^{\prime} \cos \left(V^{\prime \prime}+V^{\prime}-2 h^{\prime \prime}\right)-a^{\prime \prime} \cos 2\left(V^{\prime}-h^{\prime \prime}\right)\right\}, \\
& \zeta^{2}=2 \gamma^{\prime \prime 2} a^{\prime \prime 2}\left\{1-\cos 2\left(V^{\prime \prime}-h^{\prime \prime}\right)\right\} \text {. }
\end{aligned}
$$


Let

$$
D^{-n} a^{\prime n}=A_{0}^{(n)}+2 A_{1}^{(n)} \cos \left(V^{\prime \prime}-V^{\prime}\right)+2 A_{2}^{(n)} \cos 2\left(V^{\prime \prime}-V^{\prime}\right)+\cdots
$$

Then

$$
\begin{aligned}
& H=\frac{\lambda}{m^{\prime}}\left(A_{0}^{(3)}-6 \gamma^{\prime \prime 2} \frac{a^{\prime \prime 2}}{a^{\prime 2}} A_{0}^{(5)}\right), \\
& K=\frac{\lambda}{m^{\prime}}\left(A_{0}^{(5)}-2\left(1-\gamma^{\prime \prime 2}\right) \frac{a^{\prime \prime}}{a^{\prime}} A_{1}^{(5)}+\left(1-2 \gamma^{\prime \prime 2}\right) \frac{a^{\prime \prime 2}}{a^{\prime 2}} A_{2}^{(5)}\right) .
\end{aligned}
$$

The following table gives the values of the various quantities:

\begin{tabular}{|c|c|c|c|c|c|}
\hline Planet. & Mercury. & Venus. & Mars. & Jupiter. & Saturn. \\
\hline $\begin{array}{c}m^{\prime} \\
\lambda\end{array}$ & 6000000 & 408000 & 3093500 & 1047.35 & 3501.6 \\
\hline $\log \frac{a^{\prime \prime}}{a^{\prime}}$ & 9.5878 & 9.85934 & .1829 & .71623 & .9795 \\
\hline$A_{0}^{(3)}$ & 1.4359 & 4.9317 & 1.0858 & $10^{-3} \times 7.7267$ & $10^{-3} \times 1.181$ \\
\hline$A_{0}^{(5)}$ & 2.5658 & 41.9340 & 2.3140 & $10^{-4} \times 3.3035$ & $10^{-5} \times 1.356$ \\
\hline$A_{1}^{(5)}$ & 1.9560 & 40.7575 & 2.2042 & $10^{-4} \times 1.4856$ & $10^{-6} \times 3.483$ \\
\hline$A_{2}^{(5)}$ & 1.2086 & 37.8764 & 1.9597 & $10^{-5} \times 4.8834$ & $10^{-7} \times 6.35$ \\
\hline
\end{tabular}

Of these numbers, the values of the masses are taken from the best modern determinations; the ratios of the mean distances are certain to the degree of accuracy required here; the values of $A_{i}^{(n)}$ for Venus and of $A_{0}^{(3)}$ for Mars have been calculated from the tables given by Newcomb; ${ }^{*}$ the rest of the numbers are taken from the corresponding table of Hansen. $\dagger$

It is directly seen that the inclination of Venus exerts a slight effect. For since $\gamma^{\prime \prime}=.0296$, the ratio of the second term of $H$ to the first is $1 / 43$, and the part produced by the first term is about $1^{\prime \prime}$ as we shall see in the following table. The effect on $K$ is smaller.

* Action of the Planets on the Moon, Washington Astronomical Papers, vol. 5 (1894), pp. 248-261.

† Darlegung der theoretischen Berechnung der in den Mondtafeln angewandten Störungen (Abh. 1), Abhandlungen der mathematisch-physischen Classe der Königlich Sächsischen Gesellschafteder Wissensehaften, vol. 6 (1862), p. 487. The table above is not quite consistent for the values $A_{i}^{(n)}$ for Venus and that of $A_{0}^{(3)}$ for Mars include the squares of the inclinations and eccentricities of those planets, while the other $A_{i}^{(n)}$ do not. But as all these squares are negligible, except that of the inclination of Venus, the slight inconsistency makes no difference in the results.

Trans. Am. Matb. Soc. 19 
8. The results of the calculations are as follows:

\begin{tabular}{|c|c|c|c|c|c|c|}
\hline \multirow{2}{*}{ Planet. } & \multirow{2}{*}{$10^{8} \mathrm{H}$} & \multicolumn{2}{|c|}{ Part from $H$. } & \multirow{2}{*}{$10^{8} K$} & \multicolumn{2}{|c|}{ Part from $K$. } \\
\hline & & $\delta b_{6}$ & $\delta b_{6}$ & & $\delta b_{5}$ & $\delta b_{6}$ \\
\hline Mercury, & $+\quad 24$ & $+0^{\prime \prime} .018$ & $-0^{\prime} .017$ & +20.6 & $+0^{\prime \prime} .031$ & $+0^{\prime \prime} .001$ \\
\hline Venus, & 1181 & .906 & .860 & 687.9 & 1.060 & .041 \\
\hline Mars, & 35 & .027 & .025 & 4.7 & .007 & .000 \\
\hline Jupiter, & 738 & .566 & .537 & 10.2 & .016 & .001 \\
\hline Saturn, & 34 & .026 & .025 & 0.0 & .000 & .000 \\
\hline Sums, & +2012 & $+1^{\prime \prime} .543$ & $-1^{\prime \prime} .464$ & +723.4 & $+1^{\prime \prime} .114$ & $+0^{\prime} .043$ \\
\hline
\end{tabular}

The complete annual mean motions contributed by direct planetary action are therefore

$$
\delta b_{5}=+2^{\prime \prime} .66, \quad \delta b_{6}=-1^{\prime \prime} .42 .
$$

In order to show the degree of accuracy obtained by the method, I give the parts of these contributed by $\mathrm{e}^{2}, \mathrm{k}^{2}$. They are :

$$
\begin{aligned}
& \text { in } \delta b_{5} \text {, from } H \text {, by } \mathrm{e}^{2},-0^{\prime \prime} .004 \text {; by } \mathrm{k}^{2},-0 " .025 \text {; } \\
& \text { “ “ } " K, \text { “ } \quad-0 " .005 ; “-0 " .009 \text {; } \\
& \text { " } \delta b_{6}, \text { " } H, \text { “ }-0 " .009 ; " \quad+0 " .006 \text {; } \\
& \text { “ “ “ } K, \text { “ }-0 \text { ". } 003 ; \text { “ } \quad .000 \text {. }
\end{aligned}
$$

The parts independent of $\mathrm{e}^{2}, \mathrm{k}^{2}$ have also been calculated directly by the method of my lunar theory, and the results agree exactly, as they should, with those obtained above.

(iii) Application to the action arising from the figure of the earth.

9. The disturbing function has been fully expanded by HILL.* The only terms which will produce constant portions are, in his notation,

$$
\frac{\beta_{1}}{a^{3}} \mu\left[\frac{1}{3}-2 \gamma^{2}+2 \gamma^{4}\right] \frac{a^{3}}{r^{3}}+2 \frac{\beta_{1} \mu}{a^{3}}\left[\gamma^{2}-\gamma^{4}\right] \frac{a^{3}}{r^{3}} \cos 2 \nu,
$$

or, in rectangular coördinates,

$$
\lambda R_{0}=\lambda n^{2} a^{2}\left[\frac{a^{3}}{3 r^{3}}-\frac{z^{2} a^{3}}{r^{5}}\right]_{0}, \quad \lambda=\frac{\beta_{1}}{a^{2}},
$$

where $r^{2}=x^{2}+y^{2}+z^{2}$. In this expression $\lambda$ is a small coefficient determined from observation. I shall here adopt HiLL's value $\uparrow$ for it, since for any other

* G. W. HILL, Determination of the inequalities in the moon's motion which are produced by the figure of the earth, Washington Astronomical Papers, vol. 3 (1891), p. 213.

† Ibid., p. 340 . 
value the changes to be made are quite simple; the most probable value for $\lambda$ is discussed elsewhere.*

From the results of my lunar theory, I find

$$
\begin{aligned}
\left(\frac{a^{3}}{3 r^{3}}-\frac{z^{2} a^{3}}{r^{5}}\right)_{0}=\cdots+.1287 \mathrm{e}^{2}- & 2.0060 \mathrm{k}^{2}+.0571 \mathrm{e}^{4}-1.0817 \mathrm{e}^{2} \mathrm{k}^{2} \\
& +.0448 \mathrm{k}^{4}+.0407 \mathrm{e}^{2} e^{\prime 2}-.0136 \mathrm{k}^{2} e^{\prime 2}+\cdots
\end{aligned}
$$

\begin{tabular}{|c|c|c|c|c|c|}
\hline Characteristic. & 1 & $\mathbf{e}^{2}$ & $k^{2}$ & $e^{\prime 2}$ & Sums. \\
\hline$\delta b_{5}$ & $+6^{\prime \prime} .936$ & $+0^{\prime \prime} .041$ & $-0^{\prime \prime} .118$ & $+0^{\prime \prime} .001$ & $+6^{\prime \prime} .860$ \\
\hline$\delta b_{6}$ & $-6^{\prime \prime} .400$ & $-0^{\prime \prime} .042$ & $+0^{\prime \prime} .026$ & .000 & $-6^{\prime \prime} .416$ \\
\hline
\end{tabular}

With $\lambda=10^{-7} \times 3.6864$, the results, on substituting in (3), are

The values for $\delta b_{5}, \delta b_{6}$ differ but little from those of HILL, $\dagger+6^{\prime \prime} .820$, and $-6 " .413$, obtained by Delaunay's method with a literal expansion in powers $n^{\prime} / n$. The terms of characteristic unity have also been obtained by using the method of my theory, and the results agree with those just found. $\ddagger$

HAVERFord College,

February 23, 1904.

* E. W. BRown, On the degree of accuracy of the new lunar theory and on the final values of the mean motions of the perigee and node, Monthly Notices of the Royal Astronomical Society, vol. 64 (1904), pp. 524-534.

† Ibid., p. 344.

$¥$ The complete results for the theoretical motions of the perigee and node, together with a comparison with observation, will be found in the paper On the degree, etc., to which reference has just been made. 